\title{
APPLICATION OF D-K ITERATION TECHNIQUE BASED ON Hळ ROBUST CONTROL THEORY FOR POWER SYSTEM STABILIZER DESIGN
}

\author{
Amitava $\mathrm{Sil}^{1}$ and $\mathrm{S} \mathrm{Paul}^{2}$ \\ ${ }^{1}$ Department of Electrical \& Electronics Engineering, Neotia Institute of \\ Technology, Management And Science (Formerly Institute of Technology \& \\ Marine Engineering), Diamond Harbor, W.B, India \\ amsxii@yahoo.co.in \\ ${ }^{2}$ Department of Electrical Engineering, Jadavpur University, Kolkata, India \\ speejupow@yahoo.co.in
}

\begin{abstract}
The D-K iteration method of controller design combines $H \infty$ synthesis that ensure robustness and $\mu$-analysis that takes care of parametric uncertainties, and yields a good performance of the robust controller. The idea is basically to find a controller that minimizes the peak value over frequency of the upper bound on $\mu$. This robust control technique is applied for the Power System Stabilizer design for a Single Machine Infinite Bus (SMIB) system, which has already proved to be a successful tool in mitigating low frequency oscillations caused by small disturbances and for improving damping characteristic of a power system. Robust control toolbox of MATLAB has been used for the design. The effectiveness of the proposed PSS for wide range of operating conditions has been shown for the SMIB system with the help of simulation with MATLAB.
\end{abstract}

\section{KEYWORDS}

$H \infty$ Control theory, robustness, D-K Iteration, Power System Stabilizer, stability

\section{INTRODUCTION}

The improvement of control technique always depends on the improvement of control theory [1], [2], which has treaded the path from Conventional to Modern to Robust. Robustness has become a critical issue in present day control due to increasing complexity of physical systems under control of which power system is also no exception. Ho robust control theory [3] [4] [5] explicitly addresses the robustness issue in feedback control for Multi Input Multi Output (MIMO) as well as Single Input Single Output (SISO) system. Robust control refers to the control of plants with unknown dynamics subject to unknown disturbances. The key issue with robust control system is uncertainty. Many classical control objectives such as disturbance attenuation, robust stabilization of uncertain systems or shaping of open loop response can be expressed in terms of $\mathrm{H} \infty$ performance and tackled by $\mathrm{H} \infty$ technique [6] [7].

The Ho design techniques are closely related to the problem of minimizing the $\infty$-norm of a combination of closed loop transfer function of a feedback system. Ho Norm is equivalent to the maximum root mean square or rms energy gain of the system and is convenient for 
representing unstructured model uncertainty. The $\mathrm{H} \infty$ Norm of a scalar transfer function $G(s)$ is simply peak value of $G|j \omega|$ as a function of frequency, i.e.

$$
\|G(s)\|_{\infty}=\max _{\omega}|G(j \omega)|=\lim _{p \rightarrow \infty}\left(\int_{-\infty}^{\infty}|G(j \omega)|^{P} d \omega\right)^{1 / p}
$$

For Multi Input Multi Output (MIMO) system, in defining $\mathrm{H} \infty$ norm of $G(s)$, the peak value is replaced by the singular value, sometimes called the principal values or principal gains and the $\mathrm{H} \infty$ norm is given by

$$
\|G(s)\|_{\infty}=\max _{\omega} \bar{\sigma}|G(j \omega)|
$$

The origin of uncertainty is of manifold; due to presence of error in determination of plant parameters, change in plant parameters due to change in operating condition, the presence of error in the measurement devices used for determination of parameters, omission of certain dynamics and reduction of plant order model for a simulation test. The uncertainty can be parametric [structured] or non-parametric [un-structured / dynamic]. The unstructured uncertainty representations are used in describing un-modelled or neglected system dynamics. Those complex uncertainties usually perform at the high frequency range. However, parametric uncertainties affect the low frequency range performance and can be represented by variations of certain system parameters over some possible ranges (complex or real). Representation of uncertainty, which is the mechanism to express the difference between the model and reality, primarily varies in terms of the amount of structure they contain. Unstructured uncertainty can be represented by additive perturbation configuration, input multiplicative or output multiplicative perturbation configuration. Any plant model with uncertainty can be represented by

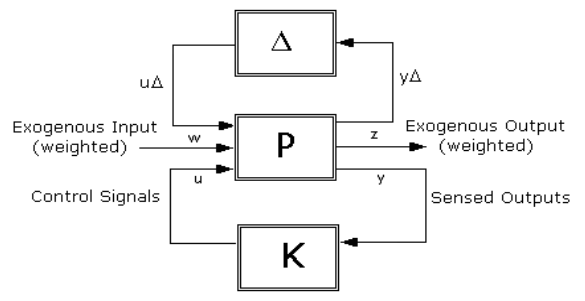

Figure 1. General Control Configuration with Model Uncertainty

where, each source of uncertainty element block $\Delta_{i}$ in $\Delta$ which is normalized such that $\left\|\Delta_{i}\right\| \leq 1$ and $\Delta_{i}$ may result from parametric uncertainty, neglected dynamics etc. Each perturbation blocks $\Delta_{i}$ are arranged in block diagonal form, to make $\Delta$ a block diagonal matrix.

The general feedback control system as shown in Fig.1 is transformed into the $\mathrm{M}-\Delta$ structure shown by the block diagram in Fig. 2, where the lower loop with controller $\mathrm{K}$ is drawn into the $\mathrm{M}$ block along with the plant $P$.

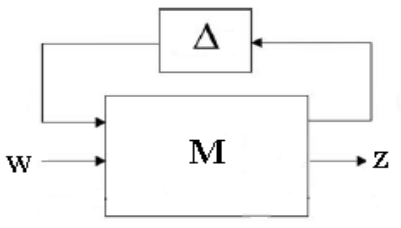

Figure 2. General $\mathrm{M}-\Delta$ structure 
The interconnection transfer function matrix $\mathrm{M}$ in Fig. 2 can be partitioned as under:

$M(s)=\left[\begin{array}{ll}M_{11} & M_{12} \\ M_{21} & M_{22}\end{array}\right]$

This gives $z=\left[M_{22}+M_{21} \Delta\left(I-M_{11} \Delta\right)^{-1} M_{12}\right] w$, if $\left(I-M_{11} \Delta\right)^{-1}$ exists.

Let $F(M, \Delta)=M_{22}+M_{21} \Delta\left(I-M_{11} \Delta\right)^{-1} M_{12}$. The Structured Singular Value (SSV) or $\mu$ analysis is an effective tool in dealing with robust analysis problem of a controller where the model uncertainties are represented in a structured manner [8], [9].

The plant matrix $\mathrm{P}$ in Fig. 1 neither includes controller $\mathrm{K}$, nor any uncertainty block $\Delta$ and $\mathrm{P}$ can be partitioned to match with 3 - input $[w, u, u \Delta]$ and 3 - output $[z, y, y \Delta]$ as

$$
P=\left[\begin{array}{lll}
P_{11} & P_{12} & P_{13} \\
P_{21} & P_{22} & P_{23} \\
P_{31} & P_{32} & P_{33}
\end{array}\right]
$$

In the $\mathrm{D}-\mathrm{K}$ iteration method of controller design, a general practice is to test robust performance in the case of input multiplicative uncertainty as it occurs physically with performance defined in terms of weighted sensitivity ' $\mathrm{W}_{\mathrm{P}} \mathrm{S}$ ', where $\mathrm{W}_{\mathrm{P}}$ is the performance weight and $\mathrm{S}$ represents the set of perturbed sensitivity functions.

This D-K iteration method of controller design, which combines $\mathrm{H} \infty$ synthesis that ensure robustness and $\mu$-analysis that takes care of parametric uncertainties, and yields a good performance of the robust controller has been applied for the Power System Stabilizer (PSS) design [10], [11]. PSS contributes significantly in mitigating the spontaneous low frequency oscillations in the range between 0.2 to $3 \mathrm{~Hz}$, which power systems experiences, by generating supplementary control signal. Then $F(M, \Delta)$ is a Linear Fractional Transformations [LFT] of M and $\Delta$. Because the "upper" loop of $\mathrm{M}$ is closed by the block $\Delta$, this kind of linear fractional transformation is also called an upper linear fractional transformation (ULFT), and denoted with a subscript u, i.e., $F_{u}(M, \Delta)$, to show the way of connection.

At times, the uncertainties include both parameter variations i.e. parametric uncertainties and unmodelled dynamics i.e. non-parametric uncertainties. All those uncertain parts still can be pulled out from the dynamics and the whole system can be rearranged in a standard configuration of (upper) linear fractional transformation $F_{u}(M, \Delta)$.

With the basic configuration of the feedback systems having $\mathrm{P}$ as the generalized plant with two sets of inputs: the exogenous inputs $w$, which include disturbances and commands, and control inputs $\mathrm{u}$ and two sets of outputs: the measured (or sensor) outputs $\mathrm{y}$ and the regulated outputs $\mathrm{z}, \mathrm{a}$ stabilizing controller $\mathrm{K}$ can be designed in presence of norm bounded specific source of uncertainty e.g. input uncertainty or parametric uncertainty and by pulling out such uncertainties into a block diagonal matrix $\Delta$ having perturbation inputs and outputs based on Structured Singular Value (SSV) denoted by $\mu$. 


\section{SYSTEM INVESTIGATED}

A single generator, 3-bus system as shown in fig. 3 is considered. The generator is having static excitation system. The nominal parameters and operating conditions are given in Appendix-A.

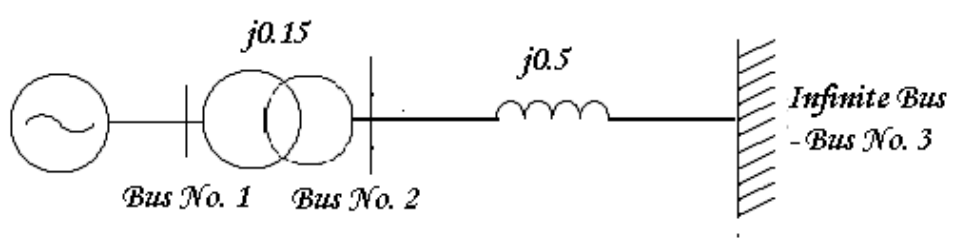

Figure 3. One line diagram of a single machine 3-bus power system

The system in general can be described by a set of coupled differential - algebraic equations, which are of the following from:

$\dot{\mathrm{x}}=\mathrm{f}(\mathrm{x}, \mathrm{z}, \mathrm{u})$

$0=\mathrm{g}(\mathrm{x}, \mathrm{z})$

where, $\mathrm{x}$ is the vector of state variables, $\mathrm{z}$ is the vector of network (algebraic) variables and $\mathrm{u}$ is the vector of control inputs. Linearization of the above set of equations followed by elimination of the algebraic variables, the above set of equations take the following state space form:

$$
\begin{aligned}
\Delta \dot{\mathrm{x}} & =\mathrm{A} \Delta \mathrm{x}+\mathrm{B} \Delta \mathrm{u} \\
\Delta \mathrm{y} & =\mathrm{C} \Delta \mathrm{x}
\end{aligned}
$$

where, $\mathrm{y}$ is the output vector. A, B and C are the constant matrices with appropriate dimensions. For the purpose of PSS design, the generator model considered is of $3^{\text {rd }}$ order and the static excitation system is of $1^{\text {st }}$ order. Thus the linearized state vector is given by $\Delta \dot{\mathrm{x}}=\left[\begin{array}{llll}\Delta \delta & \Delta \omega & \Delta E_{q}^{\prime} & \Delta E_{f d}\end{array}\right]^{T}$.

\section{PSS DESIGN}

The PSS has been designed taking speed deviation as input, the output vector is given by $\Delta \mathrm{y}=[\Delta \omega]$.

PSS is designed for the system with nominal operating condition. The nominal operating condition is completely defined by the value of real power $(\mathrm{P})$, the reactive power $(\mathrm{Q})$ at the generator terminal and is indicated in Appendix. The infinite bus voltage is $1.0 \mathrm{p}$. $\mathrm{u}$ for all loading conditions.

According to the requirement of performance constraints on control actions, the weighting function $\mathrm{W}_{\mathrm{P}}$ is added at the output. Selecting $\mathrm{W}_{\mathrm{P}}$ and $\mathrm{W}_{\mathrm{I}}$ suitably, the PSS is designed by isolating the uncertainties from the nominal plant model. Finally, PSS has been designed with $\mathrm{W}_{\mathrm{P}}$ and $\mathrm{W}_{\mathrm{I}}$ are as follows:

$$
W_{P}=\frac{1.25(s+0.05)}{s+0.001} \quad W_{I}=\frac{s+0.2}{0.5 s+1}
$$


The PSS in state space form is as follows:

$$
\begin{aligned}
& \Delta \dot{\mathrm{x}}_{\text {con }}=\mathrm{A}_{\text {con }} \Delta \mathrm{x}_{\text {con }}+\mathrm{B}_{\text {con }} \Delta \mathrm{u}_{\text {con }} \\
& \Delta \mathrm{y}_{\text {con }}=\mathrm{C}_{\text {con }} \Delta \mathrm{x}_{\text {con }}+\mathrm{D}_{\text {con }} \Delta \mathrm{u}_{\text {con }}, \text { where } \Delta \mathrm{u}_{\text {con }}=\Delta \omega \text { and } \Delta \mathrm{y}_{\text {con }}=\Delta \mathrm{u}
\end{aligned}
$$

One of the major problems of controller design using $\mathrm{H} \infty$ approaches is that the resulting controller is of a very high order, higher than the order of the original plant to be controlled. This could make such controllers impractical for large system applications. Hankel optimal model order reduction technique based on Hankel singular values, which define the "energy" of each state in the system, is applied for the reduction of the controller model [11].

\section{Simulation RESUlts}

To investigate the effectiveness of the proposed PSS the response of the power system shown in fig. 3 is simulated with and without the PSS installed. While doing the simulation studies following system conditions are considered:

i) When there is no PSS and system is having nominal load

ii) System with nominal load condition and PSS installed

iii) System with off-nominal load condition and PSS installed.

The simulation results are shown below:

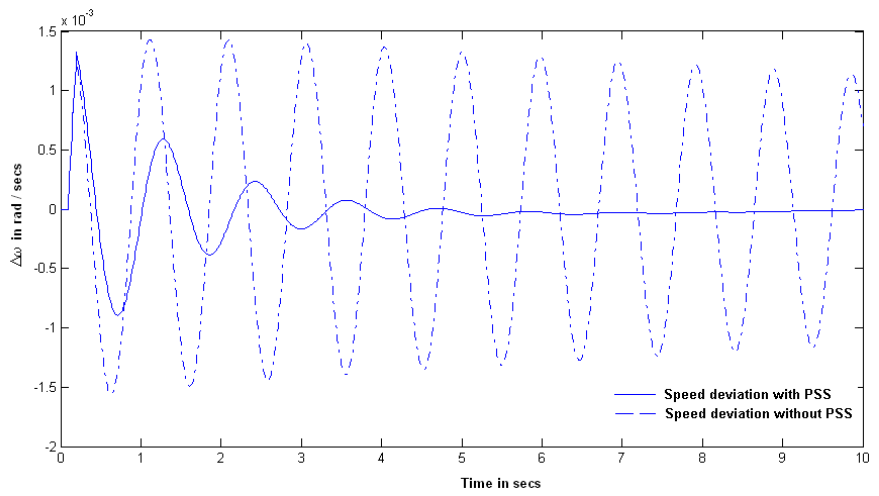

Figure 4. Comparative Result with and without PSS and System load is nominal

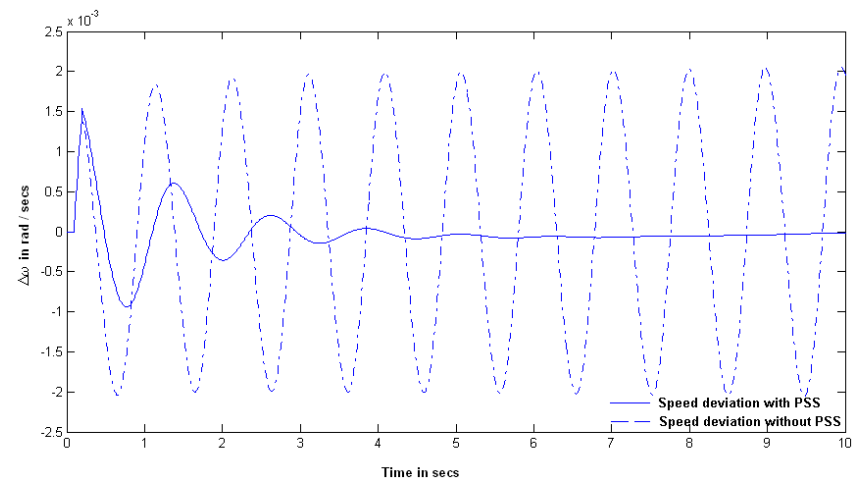

Figure 5. Comparative Result with and without PSS and System load is off-nominal 


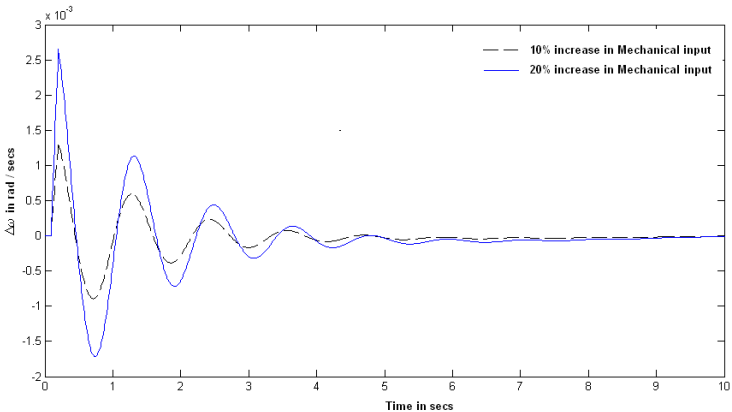

Figure 6. Comparative Result - PSS installed and Mechanical input increased by $10 \%$ and $20 \%$ for 0.10 sec. (System load is nominal)

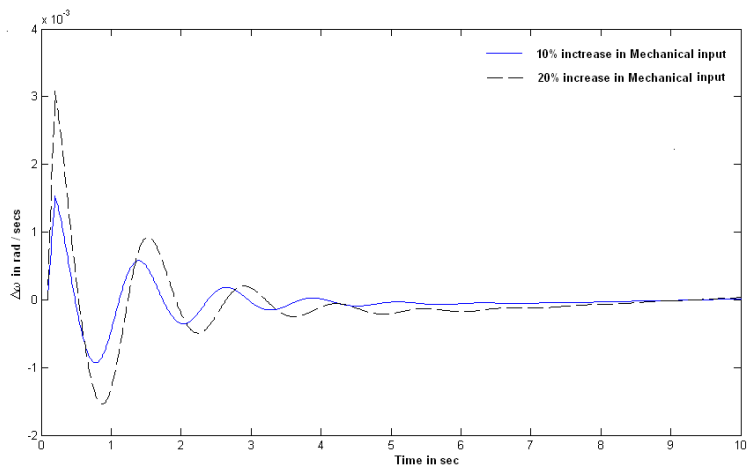

Figure 7. Comparative Result - PSS installed and Mechanical input increased by $10 \%$ and $20 \%$ for 0.10 sec. (System load is off-nominal)

The comparative simulation results in Fig. 4 and 5 indicates that there exists monotonous low frequency oscillations in the system when it is perturbed by applying disturbance in the form of $10 \%$ step increase in mechanical input for a period of 0.10 second both for nominal load and offnominal load, which damps out quite satisfactorily with the introduction of PSS both for nominal load and off-nominal load.

Simulation results for $10 \%$ step increase in mechanical input and 20\% step increase in mechanical input for 0.10 second to the machine with PSS has been plotted simultaneously for both nominal and off-nominal load and are indicated in Fig. 6 and 7. It is evident from the simulation results that the system response remains almost similar.

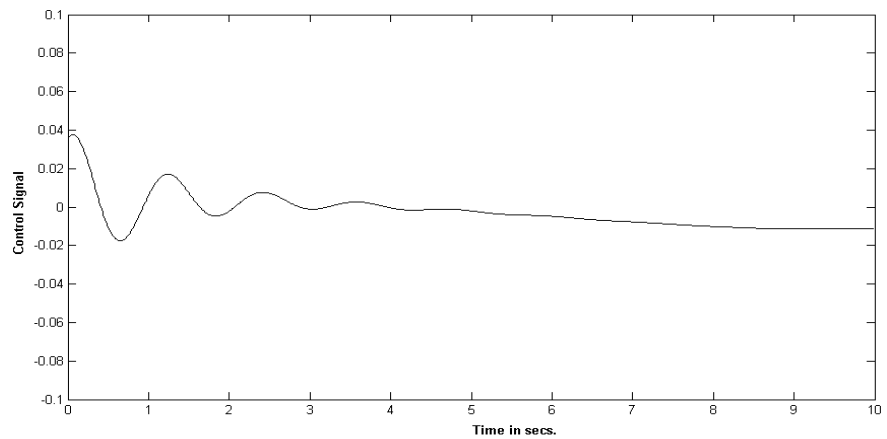

Figure 8. Plot of Control Signal with time for nominal system load 
Plot of control signal in Fig. 8, which is the output of the PSS with time, indicates that the control action ceases once the system oscillation die out following the perturbations with the introduction of Power System Stabilizer.

\section{CONCLUSIONS}

In this paper, D-K Iteration Technique based on $\mathrm{H} \infty$ Robust Control Theory has been applied for design of PSS to mitigate low frequency oscillations. The D-K iteration based controller design is based on Structured Singular Value (SSV) which may be defined as an inverse robust stability margin $\alpha_{\max }$ of the system with respect to uncertainty $\Delta$ having a block diagonal structure or $\mu$ analysis, is an effective tool in dealing with robust analysis problem of a controller where the model uncertainties are represented in a structured manner. The reduction in the controller complexity is averted by reducing controller order keeping in view suitability for practical installation. It may be concluded that application of this robust control technique for controller design (PSS here) can improve the damping of low frequency power system oscillation through a wide range of operating conditions without changing the parameters of the PSS. Simulation studies have confirmed the performance of the designed PSS has not suffered when the system operating condition has changed.

\section{APPENDIX}

The nominal parameters and the operating conditions of the system are presented below:

$$
\begin{aligned}
& \mathrm{X}_{\mathrm{d}}=1.810 \text { p.u } \quad \mathrm{X}_{\mathrm{d}}^{\prime}=0.300 \text { p.u } \quad \mathrm{X}_{\mathrm{q}}=1.760 \text { p.u } \quad \mathrm{X}_{\mathrm{q}}^{\prime}=0.300 \text { p.u } \\
& \mathrm{X}_{\mathrm{d}}{ }^{\prime \prime}=0.300 \text { p.u } \quad \mathrm{X}_{\mathrm{q}}{ }^{\prime \prime}=1.760 \text { p.u } \quad \mathrm{X}_{\mathrm{l}}=0.160 \text { p.u } \quad \mathrm{R}_{\mathrm{a}}=0.003 \text { p.u } \\
& \mathrm{T}^{\prime}{ }_{\mathrm{d} 0}=9.0 \mathrm{~s} \quad \mathrm{~K}_{\mathrm{D}}=0 \quad \omega=377 \mathrm{rad} / \mathrm{s} \quad \mathrm{K}_{\mathrm{A}}=5 \mathrm{p} . \mathrm{u} \quad \mathrm{T}_{\mathrm{A}}=0.02 \mathrm{~s} \\
& \mathrm{~K}_{\mathrm{E}}=1 \text { p.u } \quad \mathrm{T}_{\mathrm{E}}=0
\end{aligned}
$$

Nominal / Light Load: $\mathrm{P}=0.90 \quad \mathrm{Q}=0.436 ; \quad$ Off Nominal / Heavy Load: $\mathrm{P}=1.03 \quad \mathrm{Q}=$ 0.345

\section{REFERENCES}

[1] Ogata Katsushiko - Modern Control Engineering (Prentice Hall of India)

[2] Kuo C Benjamin - Automatic Control Systems (Prentice Hall of India)

[3] Robust Control Theory - Carnegie Mellon University, 18-849b Dependable Embedded Systems Spring 1999, Author: Leo Rollins

[4] Skogestad Sigurd \& Postlethwaite Ian - Multivariable Feedback Control Analysis and Design (John Wiley \& Sons, Ltd)

[5] Kemin Zhou, John C. Doyle and Keith Glover - Robust and Optimal Control (PRENTICE HALL, Englewood Cliffs, New Jersey 07632

[6] Chilali Mahmoud and Gahinet Pascal - Hळ Design with Pole Placement Constraints: An LMI Approach - IEEE Transactions On Automatic Control, Vol. 41, NO. 3, March 1996, pp. 358 - 367

[7] B. Chaudhuri, B. C. Pal, A C. Zolotas, I. M. Jaimoukha and T. C. Green - Mixed-Sensitivity Approach to $\mathrm{H} \infty$ Control of Power System Oscillations Employing Multiple FACTS Devices - IEEE Trans. Power Systems, vol. 18, No. 3, August 2003 pp. 1149-1156.

[8] Lind Rick and Balas Gary J - Evaluating D - K Iteration for Control Design, Proceedings of the American Control Conference, Baltimore, Maryland June 1994 [Authorized licensed use limited to: JADAVPUR UNIVERSITY. Downloaded from IEEE Xplore] 
[9] R. Castellanosa, A.R. Messinab and H. Sarmientoa - Robust stability analysis of large power systems using the structured singular value theory - Electrical Power and Energy Systems 27 (2005) pp. 389397

[10] A. Feliachi , Zhang X., Sims C. S., “ Power system stabilizers design using optimal reduced order models, part II: design’, IEEE Trans. on Power Systems, Vol.3, no. 4, 1988, pp. 1676-1681

[11] The Math Works, Inc. $\square$-Analysis and Synthesis Toolbox, June 1998.

[12] Gary J. Balas, John C. Doyle, Keith Glover, Andy Packard, and Roy Smith. Robust Control Toolbox, The Math Works, Inc., June 2001.

\section{Authors}

Amitava Sil is Associate Professor with the Department of Electrical and Electronics Engg, at NITMAS (formerly ITME), Diamond Harbor, 24 Pgs. (S), West Bengal, India. Sil has obtained his B.E in Electrical Engg. from BESUS, Sibpore, Howrah, W.B and obtained Ph.d from Jadavpur University. His area of work power system dynamics and control.

Subrata Paul is Professor with the Department of Electrical Engineering at Jadavpur University, kolkata (West Bengal), India. His work has been primarily concerned with power system analysis, transient stability and FACTS applications
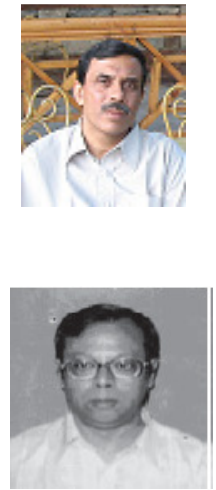